\title{
RELEVANCE FOR THE CLASSICAL LOGICIAN
}

\author{
ETHAN BRAUER
}

\author{
Department of Philosophy, The Ohio State University
}

\begin{abstract}
Although much technical and philosophical attention has been given to relevance logics, the notion of relevance itself is generally left at an intuitive level. It is difficult to find in the literature an explicit account of relevance in formal reasoning. In this article I offer a formal explication of the notion of relevance in deductive logic and argue that this notion has an interesting place in the study of classical logic. The main idea is that a premise is relevant to an argument when it contributes to the validity of that argument. I then argue that the sequents which best embody this ideal of relevance are the so-called perfect sequents-that is, sequents which are valid but have no proper subsequents that are valid. Church's theorem entails that there is no recursively axiomatizable proof-system that proves all and only the perfect sequents, so the project that emerges from studying perfection in classical logic is not one of finding a perfect subsystem of classical logic, but is rather a comparative study of classifying subsystems of classical logic according to how well they approximate the ideal of perfection.
\end{abstract}

The idea that relevance is one criterion for evaluating arguments is intuitive and compelling. The development of this idea bifurcates into informal logic in the analysis of informal fallacies, and formal logic in the development of relevance logics. In informal logic little attention is paid to the formal features of an argument, and whether the informal notion of relevance has either a formal component or a formal counterpart is generally not asked. On the formal side, despite the great technical and philosophical attention given to relevance logics, the notion of relevance itself is often left at an intuitive level. It is difficult to find an explicit account of what relevance is, leading some critics to charge that there is no coherent notion of relevance underlying relevance logic. ${ }^{1}$ Relevance logics are also typically offered as competitors to classical logic, presumably under the assumption that the study of relevance is outside the purview of classical logic. ${ }^{2}$

I believe that both the assumption that relevance is outside the purview of classical logic and the objection that there is no coherent notion of relevance are mistaken. I will

Received: April 2, 2018

2010 Mathematics Subject Classification: 03A05.

Key words and phrases: relevance, perfect sequents, classical logic.

1 E.g., Diaz (1981), Burgess (1981), Copeland (1980).

2 One notable exception here is Copeland (1984). Tennant (1979) and Makinson (2017) hold the neighboring position that strictures of relevance should not require new semantics beyond the classical methods, but rather should be manifested by more exigent syntactic requirements in proof theory. As a result, they both advocate for particular systems of relevance logic. Also notable is the position of Verdée \& de Bal (2015), who maintain that there is a concept of relevant implication which is a subconcept of classical entailment. This is similar to the view developed in §2-\$4. Their project differs in two main respects from mine: first, they characterize the notion of relevant implication by giving (philosophically motivated) technical requirements for relevant implication rather than explicating an intuitive concept, and second their theory treats relevant implication as an object-language connective. 
argue that there is an interesting notion of relevance which can be precisely defined and which is available for the classical logician to study. Beginning with the intuitive notion of a proposition bearing on a question, I offer a formal explication of the notion of relevance in deductive logic, and propose a set of research questions stemming from this notion. In $\$ 1$ I discuss some common motivations for studying relevance logics and identify two criteria an account of relevance should satisfy. In §2 I refine the intuitive notion of relevance and offer a formally precise explication of it in terms of a premise contributing to the validity of an argument. In $\$ 3$ I highlight a decision point that arises from my explication concerning what the ideal standard of relevance is, arguing that the notion of relevance is best embodied by the so-called perfect sequents-that is, sequents which are valid but none of whose proper subsequents is valid. This is primarily motivated by the intuition that nothing can further bear on a question that has already been settled, but I argue that it also has theoretical advantages. $\$ 4$ proposes a research program in proof theory that arises out of the claims of $\$ 3$. $\$ 5$ takes up the comparison of my account of relevance with other approaches to relevance logic, and $\S 6$ is a conclusion. A companion article begins the technical investigation of the project proposed here.

\$1. Why relevance? In this section I sketch the most common motivations for trying to account for relevance in formal logic. These take the form of certain paradoxes (or perhaps 'paradoxes') where innocent-looking logical principles lead us classify some sentences as collectively entailing another sentence, even though, intuitively, they do not entail that sentence. While I agree that there is indeed something intuitively puzzling about these examples, my goal here is not to argue for the need for a relevance logic on their basis. The goal is rather to get clear on what motivates the study of relevance in formal logic and what an appeal to relevance is hoped to achieve.

For our first example, consider the following argument schema, generally attributed to C.I. Lewis: ${ }^{3}$

1. $A$

Assumption

2. $A \vee B$ from (1)

3. $\neg A$

Assumption

4. $B$ from (2) and (3)

In what follows I will refer to this as the Lewis argument or Lewis paradox. It is of course a proof of the entailment $A, \neg A \therefore B$. The steps involved seem logically unimpeachable - they are only disjunction introduction, disjunctive syllogism, and the assumption that deducibility is transitive. And yet, it is natural to think that neither $A$ nor $\neg A$, nor even their combination has anything to do with whether $B$ holds. Thus, for instance, we find logicians making the following claims:

- "We regard a contradiction $A \wedge \neg A$ as in general irrelevant to an arbitrary proposition $B$, and we accordingly think of the principle ' $(A$ and not- $A)$ implies $B$ ' as embodying a fallacy of relevance” (Anderson \& Belnap, 1962, p. 9).

3 Cf. (Lewis \& Langford, 1959, pp. 248-251). This argument was actually known to medieval logicians; (Anderson \& Belnap, 1975, pp. 163-165) sketch a brief history. 
- "We agree with those who find the argument from $[A \wedge \neg A]$ to $[B]$ self-evidently preposterous" (Anderson \& Belnap, 1962, p. 18).

- " $B$ does not follow from $A \wedge \neg A$... If pressed on why [this inference is] rejected, the response will be along the lines that the premise 'has nothing to do with' the conclusion" (Lapara, 1976, p. 91).

- "One common diagnosis of the flaw in Lewis' analysis [of entailment] is that it allows for cases where entailment holds between irrelevant propositions. Doubtless this is so, and intuitively, one might easily think that it is irrelevance that accounts for the radical falsehood of Lewis' paradoxes" (Woods, 1964, p. 130).

Even if the argument $A, \neg A \therefore B$ is not quite 'self-evidently preposterous', something does seem peculiar about it. It is natural to ask, then, if we can cash out this notion of relevance which seemingly causes some people to balk at this argument.

The argument dual to $A, \neg A \therefore B$ is of course $B \therefore A \vee \neg A$, which can feel similarly surprising for ostensibly similar reasons. Imagine someone saying in conversation: 'I had eggs for breakfast, therefore Goldbach's conjecture is either true or false.' It would be natural to respond that of course Goldbach's is either true or false (bracketing anti-realist worries), but what does one's breakfast have to do with it? Despite being a valid argument in the Tarskian sense, it seems to fail a general standard of relevance. The premise is just unrelated to the conclusion.

In addition to these curious Lewisian arguments, the so-called paradoxes of strict implication and paradoxes of material implication are also sometimes said to embody fallacies of relevance. The paradoxes of strict implication include the sentential versions of the Lewis arguments, namely $(A \wedge \neg A) \rightarrow B$ and $B \rightarrow(A \vee \neg A)$, as well as others such as $A \rightarrow(B \rightarrow B)$. These are all valid when the arrow is read as a strict conditional. If the strict conditional is offered as an analysis of the pretheoretic concept of entailment, these do indeed seem to pose problems for that analysis. Does $A$ really intuitively entail the fact that $B$ entails itself? "Relevance logicians claim that what is unsettling about these socalled paradoxes is that in each of them the antecedent seems irrelevant to the consequent" (Mares, 2014).

When we read the arrow in these sentences as the material conditional rather than as a strict conditional, they are included among the paradoxes of material implication, along with $A \rightarrow(B \rightarrow A)$, which is the "archetype of fallacies of relevance" (Anderson $\&$ Belnap, 1975, p. 30). Again, this might seem surprising if the material conditional is supposed to be an analysis of some pretheoretic notion of entailment.

In this article, however, I will take the problem of relevance, as it applies in formal logic, to be embodied by the first two Lewis arguments rather than the paradoxes of strict or material implication. One reason for this is that strict implication is not a plausible analysis of logical entailment to begin with, and material implication is even worse. Moreover, my goal in this article is to explicate a notion of relevance in the context of classical logic, and classical logic is not generally taken to have any object-language connective that expresses the relation of logical entailment. ${ }^{4}$ If the meaning of the material conditional is kept in mind, one need not be bothered by the paradoxes of material implication, and since $A \rightarrow B$ is classically equivalent to $\neg A \vee B$, the arrow can even be omitted from the language entirely. Indeed, in proof theory it is often more convenient to take $\neg, \vee$, and $\wedge$ as the only

4 At most, the material conditional indicates entailment, cf. Meyer (1971). Whether people historically thought of the conditional as expressing entailment I am not in a position to say, though Frege certainly did not (cf. Frege, 1879, §5). 
propositional connectives. ${ }^{5}$ So if we want to find a place for relevance in classical logic, we should not look to arrows or hooks, but to entailment understood as a relation between sets of sentences.

The problem I am addressing here, then, is that some inferences, although typically taken to be valid, seem to flout norms of relevance in reasoning. What that notion of relevance actually comes to in formal logic is unclear though. My aim is to give an account of relevance, as it applies in classical logic, that can explain what is irrelevant in the Lewis arguments. There are two criteria that the resulting account of relevance should meet. First, the connection between the intuitive concept of relevance and the formal explication should be clear. This is the piece that many extant accounts of relevance are missing. While there might be something interesting to say about how an intuitive notion of relevance could be explicated in terms of relevance domains (Avron, 1984), variable sharing (Anderson \& Belnap, 1975; Avron, 2014; Tennant, 2015), or usein-a-derivation (Anderson \& Belnap, 1975; Smiley, 1959), not much has been said to make these connections. The second criterion is that the logical significance of the notion of relevance should be clear. If the only account of relevance that explained the peculiarity of the Lewis paradoxes was cast in terms of, say, pragmatic implicature, then although it would be worth studying, it might not fall within the purview of logic as such.

\$2. Refining the intuitive concept. A theory of relevance must first address what the locus of relevance is. When we seek to give an account of relevance in reasoning, what is the object that can be relevant or irrelevant? I suggest that we see relevance as a relation between a premise and the argument it figures in. This is primarily motivated by the notion of a proposition being relevant to or bearing on a question. For example, John's being tall is relevant to the question of whether he will be a good basketball player, the presence of clouds bears on the question of what the likelihood of rain later today is, and that my hair is brown is irrelevant to, or does not bear on, the question of where my kitchen table was made. At least, this is so in most ordinary contexts. Strictly speaking, we should regard whether a proposition bears on a question as a function of context-more on this below. Although I am interested here in the notion of relevance as it applies in deduction, it is worth noting that this notion of relevance also seems to be the notion of relevance that is appealed to in confirmation theory when giving an account of what makes evidence relevant to an hypothesis (Gemes, 2007; Krämer, 2017).

This notion of relevance applies to arguments when we focus on a specific type of question, namely whether a certain proposition holds. Then a premise is relevant to an argument when the proposition expressed by that premise bears on the question of whether the conclusion is true. ${ }^{6}$ Thus we might say that a premise is irrelevant to an argument when the premise does not help us establish the truth or falsity of the conclusion. By an argument, I mean a set of premises and a conclusion, and we will identify two arguments only when they have exactly the same premises and conclusions.

5 Recall that we are limiting our attention to classical logic. The intuitionist of course needs to include the arrow as a separate connective since it is independent of the other connectives in intuitionistic logic (Prawitz, 2006, Chap. IV, Corollary 9). I comment briefly on the extension to nonclassical logics in the conclusion.

${ }^{6}$ For clarity, I will usually just say 'the premise' rather than 'the proposition expressed by the premise'. 
The notion of relevance brought out here is, I claim, an intuitive and commonsense notion with clear significance for logic. Indeed, whether a premise bears on the conclusion seems to be exactly what is at stake in arguments that commit fallacies of relevance in the pretheoretic sense of fallacy of relevance. In this connection, compare the opening remarks of Burgess (1981):

Responding to Harvey's theories about the circulation of the blood, Dr. Diafoirus argues (a) that no such theory was taught by Galen, and (b) that Harvey is not licensed to practice medicine in Paris. Plainly there is something wrong with a response of this sort.... For either or both of the allegations (a) and (b) might well be true without Harvey's theory being false. So Diafoirus' argument can serve only to divert discussion from the real question to irrelevant side-issues. The traditional term for such diversionary debating tactics is "fallacy of relevance".

Clear examples of arguments whose premises are irrelevant to their conclusions are those that commit informal fallacies, such as an ad hominem or equivocation. This suggests that in locating the property of relevance at the level of arguments we have identified the correct locus of relevance. Intuitively significant concepts in philosophy are, of course, often vague and multifaceted, so there may be other legitimate notions of relevance that are logically significant. But even if this is so, it seems safe to say that we have identified an important sense of relevance if perhaps not the univocal logically salient notion.

There are three observations we can make that will build up to our account of relevance in formal deduction. The first is that whether a proposition bears on a question typically depends on the context. For instance, in a context where it is part of the common ground that John is athletic, the fact that he is tall bears on the question of whether he is good at basketball. On the other hand, in a context where it is known that John is very uncoordinated, the fact that he is tall will not bear on the question of whether he is good at basketball, since that question is effectively answered by his being uncoordinated. In logic, the important feature of the context is the argument in which the premise occurs: whether a premise bears on the question of the conclusion's truth depends on the other premises in the argument. In most arguments, no single premise settles the question of the conclusion's truth, but they do so jointly. Whether a single premise is relevant, then, can be understood as whether it bears on the question of the conclusion's truth, in the context of the other premises on offer.

Second, since we are dealing with deductive arguments rather than inductive ones, we are not concerned merely with making the conclusion more likely, but with establishing the conclusion. Thus the aim of relevance requires the premises not merely to bear on the question of whether the conclusion is true, but to settle the question.

Third, informal arguments are often enthymematic. When we evaluate an informal argument we consider not only whether it is formally valid as it stands, but whether any lacunae can be charitably filled in an appropriate formal reconstruction. Part of recognizing that an informal argument commits a fallacy of relevance is seeing that any addition to the argument to fill the gaps either will not be antecedently plausible, or will be plausible but then suffice to establish the conclusion on their own, so that the premises we started with actually play no role in the argument. Formalizing a natural language argument may help us see what premises would suffice to make it valid, but it cannot tell us whether those premises are plausible. While evaluating the plausibility of auxiliary premises is part of what goes into evaluating whether an informal argument commits a fallacy of relevance, this aspect of relevance is filtered out when we focus on formal arguments. 
Putting these three observations together, we can present the core argument of this section. The notion of the relevance of a premise to a conclusion with which we started-namely, that of the premise bearing on whether the conclusion is true-cannot be evaluated just by considering that premise and the conclusion, but depends on the full argument in which the premise occurs. So a premise is relevant to a conclusion if, when taken together with the other premises, it bears on the truth of the conclusion. In general, bearing on the truth of the conclusion could mean just having evidential value for the question of whether the conclusion is true; but in deductive logic we have the more stringent aim of demonstrably establishing the conclusion on the basis of the premises rather than simply providing some evidence for or against it. So a premise will be relevant to the conclusion of a given argument when, taken together with the other premises, it settles the truth of the conclusion. What it takes for an argument to establish its conclusion is for it to be sound, so we might say that a premise is relevant to a conclusion when it and the other premises taken together give us a sound argument. But a relevant premise should actually do something in the argument, it shouldn't just be along for the ride. Thus, let us say that a premise is relevant in an argument when it contributes to the soundness of the argument. This is almost right, but while the validity of the argument is in the purview of formal logic, the truth of the premises is not. So when we limit our attention to formal logic, contributing to the soundness of the argument comes down to contributing to the validity of the argument. Therefore, I suggest, the formal analogue of our notion of relevance is that of contributing to the validity of the argument.

But what does it mean to contribute to the validity of an argument? As noted above, by an argument I mean a set of premises and a conclusion. In the formal setting, an argument is represented as a sequent, an ordered pair of sets of sentences $\Delta$ and $\Phi$, written $\Delta: \Phi$. For a sequent to be valid means that, for any model, if all members of $\Delta$ are true in that model then at least one member of $\Phi$ is true in the model. ${ }^{7}$ Because of the compactness theorem, we can assume that both $\Delta$ and $\Phi$ are finite or empty. A sequent with an empty premise set corresponds to an argument that proves its conclusion from no assumptions, and a sequent with an empty conclusion set corresponds to an argument that reduces the conjunction of its premises to absurdity. If we wished to cleave more closely to natural language we could add an absurdity constant $\perp$ to figure as the conclusion of a sequent that refutes its premise set, evoking Dr. Watson's famous interjection: "'Holmes,' I cried, 'this is impossible!"' (Conan Doyle, 1976, p. 518). This would require a few modifications downstream, which I will mark in footnotes, but would not affect the essential character of my proposal. Likewise, we could require that the conclusion set of a sequent be a singleton, with inessential modifications to Proposition 3.2 that will also be indicated in a footnote. The multiple-conclusion setting is, however, more technically convenient as it facilitates a proof system for classical logic with many elegant proof-theoretic properties.

With all this in mind, a natural way of understanding contributing to the validity of the argument is to say that some premise $\psi \in \Delta$ does not contribute to the validity of the sequent $\Delta: \Phi$ just in case $\Delta \backslash\{\psi\}: \Phi$ is valid. ${ }^{8}$ But this will not quite do.

7 A small number of sentences separated by commas should be read as the set consisting of those sentences. Similarly, when we have a set and a sentence separated by a comma it should be read as the union of that set with the singleton of that sentence, and it is understood that that sentence does not already occur in the set.

8 This is very similar to the notion irrelevance $_{2}$ developed in Woods (1964). As a referee has pointed out, it is also structurally similar to a definition of difference-making in grounding that has been proposed by Krämer \& Roski (2017) (inspired by the related idea of difference-making 
The main reason this proposed definition is not adequate is that it fails to account for the way that the validity of an argument depends on the conclusion as well as the premises. $A, A \rightarrow B: B$ is valid, but $A, A \rightarrow B: C$ is not. The difference is in the conclusions; one bears the right sort of relation to the premises while the other does not. We should leave room in our account of relevance to say that a conclusion is irrelevant when it fails to contribute to the validity of the argument. To make the same point another way: validity consists in the nonexistence of countermodels. So for a sentence to contribute to the validity of an argument is for it to contribute to the nonexistence of countermodels. But a countermodel to $\Delta: \phi$ is just a model of $\Delta \cup\{\neg \phi\}$. And the fact that there is no such model can be due to $\phi$ or can fail to be due to $\phi$ just as much as it can be due to $\psi$ or can fail to be due to $\psi$, for some $\psi \in \Delta$.

A second issue with the proposed definition is that sometimes a sentence $\psi \in \Delta$ does contribute to the validity of $\Delta: \Phi$, but that contribution can equally be made by another sentence $\chi \in \Delta$. For instance, consider the following sequent.

$$
P \wedge Q, P \wedge R, P \rightarrow S: S .
$$

As is easy to see, one of $P \wedge Q$ and $P \wedge R$ is required for this sequent to be valid, but we do not need both. If the fact that erasing $P \wedge Q$ still left us with a valid sequent meant that $P \wedge Q$ did not contribute to the validity of $P \wedge Q, P \wedge R, P \rightarrow S: S$, then equally neither would $P \wedge R$. And if neither $P \wedge Q$ nor $P \wedge R$ contribute to the validity of $P \wedge Q, P \wedge R, P \rightarrow S: S$, then we should be able to erase them both and still be left with a valid sequent. Obviously, this is not the case.

Accounting for these gaps in our first attempt, we can define the following notions. These definitions make use of the notion of a subsequent. $\Delta^{\prime}: \Phi^{\prime}$ is a subsequent of $\Delta: \Phi$ just in case $\Delta^{\prime} \subseteq \Delta$ and $\Phi^{\prime} \subseteq \Phi$. If at least one of these inclusions is proper, then $\Delta^{\prime}: \Phi^{\prime}$ is a proper subsequent.

DEFINITION 2.1 (Redundancy). A pair of subsequents $\Delta_{1}: \Phi_{1}$ and $\Delta_{2}: \Phi_{2}$ of $\Delta: \Phi$ are redundant in $\Delta: \Phi$ if and only if $\Delta \backslash \Delta_{1}: \Phi \backslash \Phi_{1}$ is valid, and $\Delta \backslash \Delta_{2}: \Phi \backslash \Phi_{2}$ is valid, but $\Delta \backslash\left(\Delta_{1} \cup \Delta_{2}\right): \Phi \backslash\left(\Phi_{1} \cup \Phi_{2}\right)$ is not valid. ${ }^{9}$

What this says, intuitively, is that you need the members of at least one of $\Delta_{1}: \Phi_{1}$ and $\Delta_{2}: \Phi_{2}$ to secure the validity of $\Delta: \Phi$, but given either the members of $\Delta_{1}: \Phi_{1}$ or those of $\Delta_{2}: \Phi_{2}$, the others are unnecessary. You can erase the members of one of $\Delta_{1}: \Phi_{1}$ or $\Delta_{2}: \Phi_{2}$ from $\Delta: \Phi$ and still have a valid argument, but if you take away both the argument is no longer valid. It is obvious that redundancy is symmetric and irreflexive, from which it also follows that it is not transitive. The following fact is easy to verify.

causal influences). There, a fact $f$ is said to be a difference-making partial ground for a fact $g$ if there are some facts $f_{1}, \ldots, f_{n}$ such that $f, f_{1}, \ldots, f_{n}$ are a full ground for a fact $g$ but for which $f_{1}, \ldots, f_{n}$ is not a full ground for $g$. Given the close kinship between concepts of making a difference for and being relevant to, it is not surprising that there should be this similarity. On the other hand, the objections I raise against this attempted definition of relevance in the next two paragraphs do not obviously carry over to the definition of difference-making grounds. Further comparison of the two definitions would take us too far afield, though.

9 If we require the conclusion set to be nonempty, then we modify this definition to read: Two pairs $\left\langle\Delta_{1}, \Phi_{1}\right\rangle$ and $\left\langle\Delta_{2}, \Phi_{2}\right\rangle$ whose first members are subsets of $\Delta$ and whose second members are subsets of $\Phi$ are redundant in the sequent $\Delta: \Phi$ if and only if $\Delta \backslash \Delta_{1}:\left(\Phi \backslash \Phi_{1}\right)^{*}$ is valid, and $\Delta \backslash \Delta_{2}:\left(\Phi \backslash \Phi_{2}\right)^{*}$ is valid, but $\Delta \backslash\left(\Delta_{1} \cup \Delta_{2}\right):\left(\Phi \backslash\left(\Phi_{1} \cup \Phi_{2}\right)\right)^{*}$ is not valid, where for any set $\Psi$, we let $\Psi^{*}$ denote $\Psi$ if it is nonempty and $\{\perp\}$ otherwise. 
PROpOSITION 2.2. If $\Delta_{1}: \Phi_{1}$ and $\Delta_{2}: \Phi_{2}$ are redundant in $\Delta: \Phi$, then neither $\Delta_{1} \cup \Phi_{1}$ nor $\Delta_{2} \cup \Phi_{2}$ are empty.

With the definition of redundancy in hand we can build on it in a definition of irrelevance:

DEFINITION 2.3 (Irrelevance). A nonempty subsequent $\Delta_{1}: \Phi_{1}$ of $\Delta: \Phi$ is irrelevant in $\Delta: \Phi$ if and only if $\Delta \backslash \Delta_{1}: \Phi \backslash \Phi_{1}$ is valid, but for no subsequent $\Delta_{2}: \Phi_{2}$ are $\Delta_{1}: \Phi_{1}$ and $\Delta_{2}: \Phi_{2}$ redundant in $\Delta: \Phi .{ }^{10}$

Here the basic idea is still that the members of a subsequent are irrelevant if they do not contribute to the validity of the sequent. But we have added the condition that the subsequent not be redundant in recognition that the sentences in the subsequent can contribute to the validity of a sequent even if they are not strictly needed for validity of that sequent. This happens when the reason those sentences are not needed is that there is another set of sentences that provides enough relevant information. A sequent will be called irrelevant when it has some irrelevant subsequent and relevant when none of its subsequents is irrelevant.

A simple example of a (classically) irrelevant sequent is $P: P \vee \neg P$. Here $P: \emptyset$ is irrelevant, because it is not redundant with any other subsequent and $\emptyset: P \vee \neg P$ is valid. As a less trivial example of an irrelevant sequent, consider

$$
P, Q \wedge S, Q \wedge R,(P \wedge Q) \rightarrow T,(T \wedge S) \rightarrow U: U .
$$

$Q \wedge R: \emptyset$ is irrelevant in this sequent. To see this, note that the sequent is not valid if any other sentence is removed from the premise set, but remains valid when $Q \wedge R$ is removed from the premise set.

An interesting fact is that $A, B: A$ and $A: A, B$ are irrelevant, but $A \wedge B: A$ and $A: A \vee B$ are relevant. What this shows is that relevance is sensitive not just to the truth conditions of a premise set or conclusion set, but also to its structure. This might seem odd, and admittedly whether we represent an argument as having $A$ and $B$ as two separate premises as opposed one conjunctive premise $A \wedge B$ will sometimes be a more or less arbitrary decision. For instance, if we are formalizing an English argument one of whose premises is Ali is smart and he is handsome too, there seems to be little reason to prefer either $\mathrm{Sa} \wedge \mathrm{Ha}$ or $\mathrm{Sa}, \mathrm{Ha}$. On the other hand, if the premise is stated as Ali is smart and handsome, then it does seem preferable to write $\mathrm{Sa} \wedge \mathrm{Ha}$ instead of $\mathrm{Sa}, \mathrm{Ha}$. Perhaps the clearest cases are mathematical. Some defined notions in math are essentially conjunctive. For instance, $p$ is a Mersenne prime just in case $p$ is prime and there is some $n$ such that $p=2^{n}-1$. So in formalizing a mathematical argument, one of whose premises is that $p$ is a Mersenne prime, we seem to have good reason to represent the premise as a single conjunction rather than as two separate premises.

Now that my account of relevance is on the table, let us return to the two criteria of adequacy I set for a such an account. First, I said, there should be a clear connection between an intuitive conception of relevance and the formal account, and, second, the logical significance of the notion of relevance should be clear. The logical significance of which subsequents of $\Delta: \Phi$ are needed for the validity of $\Delta: \Phi$ is fairly obvious, and the next two sections will elaborate on this point. One might, however, wonder whether

10 If we require the conclusion set to be nonempty, we modify this definition to read: A nonempty subsequent $\Delta_{1}: \Phi_{1}^{*}$ of $\Delta: \Phi$ is irrelevant in $\Delta: \Phi$ if and only if $\Delta \backslash \Delta_{1}:\left(\Phi \backslash \Phi_{1}\right)^{*}$ is valid, but for no subsequent $\Delta_{2}: \Phi_{2}^{*}$ are $\left\langle\Delta_{1}, \Phi_{1}\right\rangle$ and $\left\langle\Delta_{2}, \Phi_{2}\right\rangle$ redundant in $\Delta: \Phi$. See the previous footnote for the ${ }^{*}$ notation. 
I have retained a sufficient connection with an intuitive notion of relevance, objecting that although my account classifies the Lewis arguments as irrelevant, it does so for the wrong reasons. If the reason my account classifies the Lewis argument as irrelevant is substantively different from the intuitive reasons for classifying the argument as irrelevant, then I must have lost touch with the intuitive notion of relevance. ${ }^{11}$ This objection seems to assume that we have a single clear intuitive reason for calling the Lewis paradox irrelevant, and I am not convinced that this is so. But considering the objection in more detail will bring out some ways that my account of relevance does diverge from what we might have expected of such an account pretheoretically. So let us turn to the details of the objection.

Intuitively, the problem with the sequent $A \wedge \neg A: B$ is that $A \wedge \neg A$ does not bear on whether $B$ holds. But on my view, the problem with this sequent is that you can delete $B$ and still have a valid sequent. Put this way, I might seem to have misdiagnosed the peculiarity of the Lewis paradox. But to put it this way is to ignore the motivating details of my proposal. I have not merely claimed that the problem with the Lewis paradox is that $B$ can be deleted without affecting the validity of the sequent and left the matter there. Rather, the fact that $B$ can be deleted without rendering sequent invalid is a symptom of the fact that $B$ does not contribute to the validity of the sequent $A \wedge \neg A: B$. And the fact that $B$ does not contribute to the validity of that sequent seems to me to be good grounds for saying, in a rough and ready intuitive sense, that $B$ is irrelevant in that sequent.

It is true that my account of relevance shifts the matter from whether a premise bears on the conclusion to whether the premise bears on the validity of the argument. To this extent, my account has strayed from the intuitive notion we began with. On the other hand, I have argued that this shift is important and inevitable. Whether a premise bears on a conclusion depends on what other premises are on offer. Does $A$ bear on whether $B$ ? It depends. Can we take for granted $A \rightarrow B$ ? If so, then $A$ does bear on $B$. If not, then perhaps not. We need to ask not whether a premise bears on the conclusion, but whether the premise bears on the conclusion in the context of an entire premise set. In other words, we need to look at sequents. And how do premises bear on conclusions in the context of a sequent? By helping to make the sequent valid.

It is also true that my account will sometimes classify not only premises as irrelevant, but conclusions too, and that this is another deviation from our intuitive starting point. It is not common usage to call a conclusion irrelevant. But while it is not common usage, I do not think it stretches common usage very much either. There is nothing wrong with a theoretical account of some phenomenon leading us to see new instances of that phenomenon elsewhere, provided the theoretical account is well-motivated. The explication of relevance as contributing to the validity of a sequent is, to my mind, both natural and well-motivated, and once we have accepted this it would be ad hoc to deny that conclusions can contribute or fail to contribute to the validity of a sequent.

Thus despite these deviations from our pretheoretic expectations, it seems to me that the present account captures important aspects of an intuitive conception of relevance. That being said, I do not claim that the present account is the correct, or even uniquely best, account of relevance. Intuitive concepts often have multiple threads running through them that sometimes cleave together, sometimes pull apart. There may be other interesting accounts of relevance that capture some of the other threads of our intuitive conception of relevance. All I wish to claim is that the present account captures some of these threads as well.

11 I am indebted here to an anonymous referee. 
\$3. Relevance or perfection? Relevance, as defined above, aims to capture the notion of contributing to the validity of a sequent, and the relevant sequents can be thought of as those that are obtained from the valid sequents by weeding out the premises and conclusions that do not help make the sequent valid. If we push this idea a little further, we might also weed out premises and conclusions that are not indispensable to the validity of the sequent. This would leave us with sequents that are valid, but would become invalid if we deleted any further premise or conclusion. In other words, we would have sequents that are valid but have no valid proper subsequents: the so-called perfect sequents. ${ }^{12}$ Should relevance or perfection be the ultimate aim of a logic that respects the intuitive idea that the premises should bear on the truth of the conclusion? That is, if we wanted to develop a relevance logic to capture the idea that premises and conclusions should all bear on the validity of the argument, should that logic prove exactly the relevant sequents, or exactly the perfect sequents? In this section I will suggest that perfection is the better answer to this question (though as we will see in the next section, this is something of a false dichotomy). ${ }^{13}$

The primary motivation for taking the perfect sequents as the ideal goal of a logic that respects relevance is the intuition that nothing can further bear on a question that has already been settled. Say we have a perfect sequent $\Delta: \phi$. Then $\Delta$ settles the question of whether $\phi$. So even if $\Delta, \Gamma: \phi$ is relevant in the above sense, it would be infelicitous to say that $\Gamma$ contributes to settling the question of whether $\phi$ in the sequent $\Delta, \Gamma: \phi$, since $\phi$ is already settled by $\Delta$. We can further make the following observations.

Proposition 3.1. $\Delta: \Phi$ is relevant iff for all $\phi \in \Delta \cup \Phi$, there is some perfect subsequent $\Delta^{\prime}: \Phi^{\prime}$ such that $\phi \in \Delta^{\prime} \cup \Phi^{\prime}$.

Proof. $(\Leftarrow)$ Let $\Gamma: \Sigma$ be an arbitrary subsequent of $\Delta: \Phi$ such that $\Delta \backslash \Gamma: \Phi \backslash \Sigma$ is valid. (If there is no nonempty such subsequent, then $\Delta: \Phi$ is perfect, hence relevant). Take $\phi \in \Gamma$ (or $\phi \in \Sigma$ ), and assume that there is some perfect subsequent $\Gamma^{\prime}: \Sigma^{\prime}$ of $\Delta$ : $\Phi$ such that $\phi \in \Gamma^{\prime}$ (or $\phi \in \Sigma^{\prime}$, respectively) and $\Gamma \neq \Gamma^{\prime}$ (or $\Sigma \neq \Sigma^{\prime}$, resp.). Then $\Delta \backslash\left(\Delta \backslash \Gamma^{\prime}\right): \Phi \backslash\left(\Phi \backslash \Sigma^{\prime}\right)$ is valid, but $\Delta \backslash\left(\Gamma \cup\left(\Delta \backslash \Gamma^{\prime}\right)\right): \Phi \backslash\left(\Sigma \cup\left(\Phi \backslash \Sigma^{\prime}\right)\right)$ is not valid. So $\Gamma: \Sigma$ is redundant with $\Delta \backslash \Gamma^{\prime}: \Phi \backslash \Sigma^{\prime}$ in $\Delta: \Phi$, and hence $\Delta: \Phi$ is relevant. On the other hand, if there is no such $\Gamma^{\prime}: \Sigma^{\prime}$, then $\Gamma: \Sigma$ must be perfect, and hence is redundant with $\Delta \backslash \Gamma: \Phi \backslash \Sigma$.

12 The notion of a perfect sequent was, I believe, first introduced in Tennant (1984). If we require that the conclusion set be nonempty, then we must also stipulate that $\Delta: \perp$ is a subsequent of $\Delta: \phi$.

13 Lehrer (1973) also gives an account of relevance according to which the relevant deductive arguments are the perfect sequents, though by stipulation he excludes sequents with empty premise sets or empty conclusion sets from this class. The way he reaches this account of relevance is also different from my own: his starting point is the claim that a premise is relevant in a deductive argument if a person would have to know that premise to come to know the conclusion by that deduction. This epistemological focus of Lehrer's account makes it less flexible than my approach in extending to other logics. First, it makes sense to talk about which sequents are perfect or relevant even when they are sequents of a logic with little or no epistemological significance (e.g., a logic with no complete deductive system, such as full second-order logic). Second, it is presumably impossible to come to know some proposition by deduction in an unsound logic. So if, say, intuitionistic logic were the correct logic, one could not come to know $\phi$ by means of a strictly classical proof of $\phi$. So Lehrer's account of relevance is tied to questions of what the right logic is. See also the discussion in $\$ 5.1 \& \$ 5.2$ below. 
$\Leftrightarrow)$ By induction on $|\Delta \cup \Phi|$. Assume the claim holds for all sequents $\Lambda: \Psi$ such that $|\Lambda \cup \Psi|<|\Delta \cup \Phi|$. Let $\phi \in \Delta$ be arbitrary (the argument is similar if $\phi \in \Phi)$. If $\Delta \backslash\{\phi\}: \Phi$ is not valid, then $\phi$ is a member of every perfect subsequent of $\Delta: \Phi$. If $\Delta \backslash\{\phi\}: \Phi$ is valid, then there exists another subsequent $\Gamma: \Sigma$ of $\Delta: \Phi$ which is redundant with $\phi: \emptyset$. In particular, $\Delta \backslash \Gamma: \Phi \backslash \Sigma$ is valid and $\Delta \backslash(\Gamma, \phi): \Phi \backslash \Sigma$ is not valid. Therefore, $\phi: \varnothing$ is not irrelevant in $\Delta \backslash \Gamma: \Phi \backslash \Sigma$, so we can find some valid and relevant subsequent $\Delta^{\prime}: \Phi^{\prime}$ of $\Delta \backslash \Gamma: \Phi \backslash \Sigma$ such that $\phi \in \Delta^{\prime}$, and by the inductive hypothesis we are done.

PROPOSITION 3.2. If $\Delta: \Phi$ and $\Gamma: \Psi$ are both relevant, then $\Delta, \Gamma: \Phi, \Psi$ is relevant. $^{14}$

Proof. If $\Delta: \Phi$ and $\Gamma: \Psi$ are both relevant, then by the previous proposition any $\phi \in \Delta \cup \Phi \cup \Gamma \cup \Psi$ is a member of a perfect subsequent of either $\Delta: \Phi$ or $\Gamma: \Sigma$ (according to whether $\phi$ came from $\Delta: \Phi$ or $\Gamma: \Sigma$ ), which in either case is also a perfect subsequent of $\Delta, \Gamma: \Phi, \Psi$, so by the last proposition again, $\Delta, \Gamma: \Phi, \Psi$ is relevant.

A simple corollary is that if both $\Delta: \phi$ and $\Gamma: \phi$ are perfect, then $\Delta, \Gamma: \phi$ will be relevant. I am somewhat inclined to say that, although $\Delta, \Gamma: \phi$ is relevant, if $\Delta \neq \Gamma$ then the relevance of this sequent is not due to a genuine relation of $\Delta, \Gamma$ settling the question of whether $\phi$. Rather, it is derivative on the facts that $\Delta$ settles $\phi$ and $\Gamma$ settles $\phi$. As a concrete example, take

$$
\Delta=A, A \rightarrow C
$$

and

$$
\Gamma=B, B \rightarrow C \text {. }
$$

$\Delta: C$ and $\Gamma: C$ are both perfect, and $\Delta$ and $\Gamma$ each wholly bear on, and settle, the question of whether $C . \Delta \cup \Gamma$, by contrast, is the cobbling together of two legitimate yet distinct sets of propositions that genuinely do wholly bear on whether $C$. The perfect sequents are a more natural ending point for an explication of the intuitive notion of relevance we isolated: that of bearing on the question of whether the conclusion holds.

There are also some practical advantages to taking perfection to be the aim of a logic that satisfies the ideal of relevance. First, aiming for perfect sequents rather than merely relevant sequents allows us to draw some finer grained distinctions among logical relations. Let $\Delta: \phi$ and $\Gamma: \phi$ both be perfect. Then for $\delta \in \Delta$ or $\gamma \in \Gamma$ we can say that $\delta$ is essential in $\Delta: \phi$ and $\gamma$ in $\Gamma: \phi$. This tells us something more than merely that $\delta$ is relevant to some particular sequent with conclusion $\phi$. For instance, we could say that $\delta$ is relevant in $\Delta, \Gamma: \phi$, but this doesn't actually reveal anything about the role of $\delta$ in this argument; there could be a proof establishing that $\Delta, \Gamma \vdash \phi$ in which $\delta$ does not even appear. Moreover, since $\delta$ is relevant to some sequent with conclusion $\phi$ just in case there is a perfect subsequent containing $\delta$, specifying which sequents are perfect is the most informative way of describing $\delta$ 's relation to $\phi$.

Second, aiming for perfect sequents respects the mathematical practice of looking for a weakest set of assumptions needed to establish a theorem and/or for the strongest conclusion that can be drawn from a given set of assumptions. A perfect sequent is by definition

14 If we require the conclusion set to be at most a singleton, then we restate this proposition as: If $\Delta: \phi$ and $\Gamma: \phi$ are both relevant, then $\Delta, \Gamma: \phi$ is relevant. The proof of Proposition 3.1 holds when $\Phi$ is at most a singleton, and hence the proof of our modified Proposition 3.2 is straightforward. 
the logically strongest result that can be extracted from a given argument. ${ }^{15}$ This picture of mathematical practice is something of an idealization, since mathematicians often look for a balance between logical strength and the simplicity and readability of a theorem; a simple theorem that covers the interesting cases might be preferred to one that covers all cases but is much more difficult to prove. If we wanted to be more sensitive to actual mathematical practice, it would perhaps be better to offer a theory of approximate relevance that allowed us to determine how irrelevant or redundant a theorem was. We could then perhaps couple this with an account of the complexity of mathematical concepts, as for instance proposed by Friedman \& Flagg (1990), to compare tradeoffs of decreased relevance for more conceptual simplicity, and so forth. Fortunately, as I will argue in the next section, something along these lines falls out of the account I have given so far.

So although there is a natural distinction between relevance and redundancy as defined above, these considerations suggest that the ideal ending point in the search for a formal condition that captures the intuitive notion of a premise set bearing on whether the conclusion is true, the real target should be the notion of perfection rather than the condition of relevance defined earlier. I do not regard these considerations as definitive, though. As I have emphasized, the intuitive notion of relevance is vague, and whether it is best captured by the formal definition of relevance or of perfection is a matter of how demanding you take the intuitive concept to be. Fortunately, we need not pick relevance or perfection to the exclusion of the other. As I will argue in the next section, they both have a role to play in our study of classical logic.

§4. Perfection as a measuring stick. We have defined various notions-redundancy, relevance, and perfection-in trying to isolate a condition that captures the notion of relevance in classical logic. It remains to say something about how this notion fits into the study of classical logic. The most obvious question is whether there is a subsystem of classical logic that proves all and only the perfect sequents. There are serious obstacles to giving a well-behaved proof system that only allowed the derivation of perfect sequents. To see this, consider the inference:

$$
\frac{\phi}{\phi \vee \psi}
$$

This inference cannot be generally valid in a proof system for perfect sequents, for if $\psi$ is logically true, then $\vdash \phi \vee \psi$, and hence $\phi: \phi \vee \psi$ is not perfect. So $\vee$-introduction cannot apply unrestrictedly in a proof system for perfect logic. To make matters worse, Church's theorem says that in first-order logic (with some nonunary relation symbols) it will be undecidable whether $\psi$ is logically true. Accordingly, whether $\vee$-introduction is perfection-preserving will be undecidable. We can sharpen this observation somewhat.

PROPOSITION 4.1. There is no proof system that proves all and only the perfect sequents of first-order classical logic, and for which the relation " $\Pi$ is a proof of $\Delta: \Phi$ " is decidable.

Proof. Suppose there is such a proof system. Since proofhood is decidable, the provable sequents will be recursively enumerable (r.e.). And since the provable sequents are exactly the perfect sequents, perfection is also r.e. For any sentence $\phi$ and $A$ an atom not in $\phi$, the

15 Though this maximum in logical strength may not be unique, since a single relevant sequent may contain multiple perfect subsequents. Thanks here to Neil Tennant. 
sequent $\phi \wedge A: A$ will be perfect just in case $\phi$ is satisfiable. Thus an enumeration of the satisfiable sentences can be extracted from an enumeration of the perfect sequents, so the satisfiable sentences will be r.e. On the other hand, the sequent $\phi: \emptyset$ will be perfect just in case $\phi$ is not satisfiable, so an enumeration of the unsatisfiable sentences can be obtained from an enumeration of the perfect sequents, and hence the unsatisfiable sentences will also be r.e. But if a set and its complement are both r.e., then the set is decidable. So the unsatisfiable sentences are decidable. But this contradicts Church's theorem.

Formal proofs, as syntactic objects, should be finite objects. And ideally, one should be able to ascertain what a proof actually proves by inspecting it. Thus, it seems fair to summarize Proposition 4.1 as saying that there can be no well-behaved proof system that proves all and only the classical perfect sequents. That there is no such proof system does not mean, however, that perfection has no role to play in the study of classical logic. As an analogy, consider axiomatic theories in mathematical logic. Completeness and demonstrable consistency are ideal standards for a theory, and in some cases they can be achieved. ${ }^{16}$ Similarly, I have argued, perfection is an ideal standard for a system of logic. And in limited domains it can be achieved. In propositional logic, for instance, we can generalize the truth-table method to obtain a decision procedure for perfection: enumerate all the subsequents of a given sequent and use truth tables to determine if any of the subsequents is valid. Just as Gödel's incompleteness theorems show that if a recursive theory interprets Robinson arithmetic, it will not be complete and cannot prove its own consistency, ${ }^{17}$ so Church's theorem shows that for first-order logic there cannot be a proof system that proves all and only the perfect sequents and for which proofhood is decidable. Nevertheless, in mathematical logic, the notions of completeness and provable consistency do not lose their interest or importance-the research questions surrounding them just change. Studying the deductive power and consistency strength of a theory amount to asking how complete that theory is and what it would take to prove its consistency. Such questions form the basis of reverse mathematics and reductive proof theory.

Similarly, I am suggesting, even though perfection cannot be attained, it retains its interest as a measuring stick by which to compare various logics or fragments of a given logic. This motivates a program in structural proof theory studying the perfection of various deductive systems. How extensive are the imperfections they prove? Is there a class of formulas for which they prove only perfect sequents? What are the deductive sources of the imperfections they prove? Because relevance is on a spectrum with perfection, it falls out naturally as one of the benchmarks by which to evaluate proof systems. (This is why I said that the choice between relevance and perfection was a false dichotomy). Does a given system prove only the relevant sequents? How extensive are the irrelevancies? And what are the deductive sources of the redundancies?

While relevance and perfection are natural benchmarks on a spectrum, they are only two, and it would also be interesting to develop a more finely grained system of measurement for comparing how relevant two sequents are. An obvious measure of degrees of relevance would be how many perfect subsequents a relevant sequent has. A sequent with two perfect subsequents might be said to be more relevant than a sequent with five perfect subsequents. We can then also ask questions such as whether there is any relation between, say, the com-

16 For instance, Presburger arithmetic and the theory of algebraically closed fields are both complete, see Chang \& Keisler (2012, p. 41), Boolos, Burgess, \& Jeffrey (2007, p. 295).

17 To be more precise, if $T$ is recursive and interprets Robinson arithmetic, and $\tau$ is a $\Sigma_{1}$ definition of $T$, then $T$ cannot prove $\operatorname{Con}_{\tau}$, cf. Feferman (1960). 
plexity of propositional proofs and how relevant the sequent proved is, or what syntactic properties sequents of a given degree of relevance have. The technical questions to pursue are many and interesting.

The role of relevance in classical logic, then, is not to isolate some relevant kernel of classical logic as the correct logic, but rather to draw distinctions within classical logic, and to provide us with the tools to study more finely the proof-theoretic properties of classical logic and its subsystems. There is some similarity here to the position espoused by Tennant when, in giving an early statement of his proof-theoretic approach to entailment, he says: “"Entailment logic' ... should not be thought of as a rival alongside the usual rivals such as classical and intuitionistic logic. Rather, a general theory of entailment should produce a philosophically motivated and uniform method for extracting the entailment fragments of these" (Tennant, 1979, p. 168). I have framed the issue in terms of perfection rather than entailment, and as we have seen, we cannot expect to extract the perfect fragment of classical logic. Rather, the question is how well we can approximate to the perfect fragment of classical logic, and what the obstacles are that prevent us from doing so more exactly.

A companion article begins the investigation of the research questions raised here. In that article I establish a variable-sharing result for the perfect sequents of classical logic and study the proof system that results from the classical sequent calculus by dropping the rules of cut and weakening, and restricting initial sequents to be of the form $P: P$, for $P$ atomic. This system is shown to be sound, complete, and cut-admissible with respect to the class of perfect sequents whose logical vocabulary is among $\forall, \exists$, and at most one of $\neg, \vee$, and $\wedge$. (In the fragment based on $\wedge$, however, the completeness result only holds in the restricted form that if $\Delta: \phi$ is perfect, then $\wedge \Delta: \phi$ is provable, and likewise for the cut-admissibility result).

§5. Comparisons. In this section I compare the notion of relevance explicated here with other relevance logics and related theories of entailment that have been proposed. My primary goal here is to highlight points of similarity and difference between my account and those below, not to evaluate them. I will, however, make note of when the comparisons can help in that evaluation.

5.1. Smiley, Geach, von Wright. Smiley (1959) considers two different approaches to defining entailment so as to avoid the Lewis paradoxes, and with each approach considers two different definitions. For now, we will focus on the first approach, which is to define entailment in terms of truth preservation. To avoid ambiguity, let us use $\rightarrow_{i}$ to symbolize the different definitions of entailment. Then Smiley's first definition is (p. 240):

$\phi_{1}, \ldots, \phi_{n} \rightarrow 1 \psi$ just in case $\left(\phi_{1} \wedge \cdots \wedge \phi_{n}\right) \rightarrow \psi$ is a substitution instance of a tautology $\left(\phi_{1}^{\prime} \wedge \cdots \wedge \phi_{n}^{\prime}\right) \rightarrow \psi^{\prime}$ such that neither $\neg\left(\phi_{1}^{\prime} \wedge\right.$ $\left.\cdots \wedge \phi_{n}^{\prime}\right)$ nor $\psi^{\prime}$ are tautologies.

His second definition is more demanding (p. 243):

$\phi_{1}, \ldots, \phi_{n} \rightarrow_{2} \psi$ just in case $\left(\phi_{1} \wedge \cdots \wedge \phi_{n}\right) \rightarrow \psi$ is a tautology and neither $\neg\left(\phi_{1} \wedge \cdots \wedge \phi_{n}\right)$ nor $\psi$ are tautologies.

Smiley motivates the definition of $\rightarrow_{1}$ by first noting that there is presumably nothing wrong with the classical inferences applied to contingent premises- the Lewis argument, after all, turns essentially on appealing to an impossible collection of premises. Second, he claims that the same inferences should apply to impossible and tautologous premises 
when they are substitution instances of contingent premises (Smiley, 1959, p. 239). Even though $(A \wedge \neg A) \wedge B$ is logically impossible, it is of the same form as $C \wedge B$, and hence the legitimacy of the inference $C \wedge B \therefore B$ should also legitimate $(A \wedge \neg A) \wedge B \therefore B$. After all, deductive logic is concerned with the form of valid inferences, and these two inferences have the same form. If the goal of a theory of entailment or relevance is to construct a logic of entailment or relevance, then this motivation is compelling. But if, as I have proposed, the goal is to identify a special class of sequents and study that class within an existing logical system, then this line of reasoning is much less compelling. Why should we expect that special class of sequents to be closed under substitution in the same way that derivability is? Indeed, neither the set of perfect sequents nor the set of relevant sequents is closed under substitution, but I do not see that they are any the less interesting for this fact.

An alternative motivation for the definition of $\rightarrow 1$ is offered by Geach. He and von Wright had argued in the '50s that $\phi$ entails $\psi$ just in case there is a way of coming to know $\phi \rightarrow \psi$ that is not also a way of coming to know $\neg \phi$ or coming to know $\psi{ }^{18}$ In Geach (1970) he offered a definition equivalent to that for $\rightarrow_{1}$ as a formalization of this account of entailment. The idea is this: if neither $\neg \phi$ nor $\psi$ are tautologies, then $\phi \rightarrow \psi$ is logically true just in case there is a proof of it, and no such proof will contain a proof of either $\neg \phi$ or $\psi$. That proof would be a way of coming to know $\phi \rightarrow \psi$ that is not also a way of coming to know $\neg \phi$ or $\psi$. And if $\phi^{\prime} \rightarrow \psi^{\prime}$ is a substitution instance of $\phi \rightarrow \psi$, then the corresponding substitution instance of the proof would similarly be a way of coming to know $\phi^{\prime} \rightarrow \psi^{\prime}$ without also being a way of coming to know $\neg \phi^{\prime}$ or coming to know $\psi^{\prime}$. This is because even if $\phi^{\prime}$ is logically false or $\psi^{\prime}$ logically true, the proof only depends on structural features of $\phi^{\prime}$ and $\psi^{\prime}$ that are shared with $\phi$ and $\psi$. Since $\phi$ and $\psi$ are logically consistent, the proof depends only logical structure of $\phi^{\prime}$ and $\psi^{\prime}$ that is too coarse-grained to reveal the logical falsehood of $\phi^{\prime}$ or logical truth of $\psi^{\prime}$. This explication is fairly compelling given the von Wright/Geach account of entailment. Since, however, I began from a different starting point-in particular, one not directly tied to epistemological considerations-it is not surprising that there are differences between my proposal and the Smiley-Geach-von Wright picture.

The most notable difference between these definitions and the notions of perfection and relevance is that Smiley's definitions allow for adding irrelevant premises to an entailment. It is obvious that, provided $\phi_{0}$ is not logically false, $\phi_{0}, \phi_{1}, \ldots, \phi_{n} \rightarrow_{2} \psi$ whenever $\phi_{1}, \ldots, \phi_{n} \rightarrow_{2} \psi$. For Smiley's first definition, we may even omit the proviso that $\phi_{0}$ not be logically false. To see this, suppose $\left(\phi_{1} \wedge \cdots \wedge \phi_{n}\right) \rightarrow \psi$ is a substitution instance of a tautology $\left(\phi_{1}^{\prime} \wedge \cdots \wedge \phi_{n}^{\prime}\right) \rightarrow \psi^{\prime}$ such that neither $\neg\left(\phi_{1}^{\prime} \wedge \cdots \wedge \phi_{n}^{\prime}\right)$ nor $\psi^{\prime}$ are tautologies, and let $A$ be an atom not occurring in any of $\phi_{1}^{\prime}, \ldots, \phi_{n}^{\prime}, \psi^{\prime}$. Then for any $\phi_{0}$, $\left(\phi_{0} \wedge \phi_{1} \wedge \cdots \wedge \phi_{n}\right) \rightarrow \psi$ is a substitution instance of the tautology $\left(A \wedge \phi_{1}^{\prime} \wedge \cdots \wedge \phi_{n}^{\prime}\right) \rightarrow \psi^{\prime}$ where neither $\neg\left(A \wedge \phi_{1}^{\prime} \wedge \cdots \wedge \phi_{n}^{\prime}\right)$ nor $\psi^{\prime}$ are tautologies. So $\rightarrow_{1}$ allows the addition of arbitrary premises, and $\rightarrow_{2}$ allows the addition of contingent premises.

By contrast, no premises may be added to a perfect sequent while maintaining perfection. Relevance can preserved under the addition of premises, but only with tight constraints on those premises. Specifically, if $\Delta: \Phi$ is relevant, then $\Delta, \phi: \Phi$ will be relevant just in case there are some subsequents $\Delta_{1}: \Phi_{1}$ and $\Delta_{2}, \phi: \Phi_{2}$ which are redundant.

Aside from allowing the addition of contingent premises, $\rightarrow_{2}$ is very similar to the notion of a perfect sequent. The limiting cases of perfect sequents where either the premise set or

18 Geach (1958), von Wright (1957); Anderson \& Belnap, 1975, p. 215 called this the von WrightGeach-Smiley criterion for relevance. 
the conclusion set is empty do not fall under $\rightarrow_{2}$, but can easily be accounted for by extra conditions. The case where the conclusion set has more than one member can be accounted for easily by writing a disjunction of the conclusions in the definition of $\rightarrow_{2}$.

Substitution can easily render a perfect sequent irrelevant, though, so that $\rightarrow_{1}$ does not line up even approximately with either of our notions of perfection or relevance. For instance, $A \wedge B: B$ is perfect, but its substitution instance $A \wedge \neg A: \neg A$ is irrelevant.

5.2. Tennant. Tennant's approach to relevance logic begins with the intuition that the Lewis paradox $A, \neg A \therefore B$ is objectionably irrelevant. Rather than offering an intuitive account of what relevance is, though, he proceeds by isolating the demands we place on a relevance logic. In introducing his project in Tennant (1979), he argued for a set of requirements that a theory of entailment must meet to sustain existing practices of deductive reasoning. More recently, he has framed the project by the question:

How best might one restrict the deducibility relation of a familiar system of logic (such as intuitionistic or classical logic) so as to avoid the First Lewis Paradox, but still provide all the proofs needed for mathematics and for the hypothetico-deductive method in natural science? (Tennant, 2005, p. 696)

By combining the account of relevance developed above with the insights of Smiley and Geach, we can provide an intuitive motivation for Tennant's system of Classical Core Logic (previously known as CR) and its constructive counterpart Core Logic (previously known as IR). Suppose we take the arguments of $\S 2$ and $\S 3$ to motivate the claim that perfection is the ideal standard of relevance. But, per Smiley's insight, logic is concerned with truth-preservation in virtue of logical form, and substitution preserves logical form, so we want a notion of consequence that is closed under uniform substitution. That is, if $\Delta^{\prime}$ and $\phi^{\prime}$ are obtained from $\Delta$ and $\phi$ by uniform substitution, and $\Delta$ entails $\phi$, then $\Delta^{\prime}$ should entail $\phi^{\prime}$. So if we begin with the perfect sequents and close under uniform substitution, we will get as the class of relevant entailments the so-called perfectible sequents, that is, the substitution instances of perfect sequences. And per Geach's insight, although some entailments with inconsistent premise sets will obtain, those of entailments will never turn on the inconsistency of the premise set. Classical Core Logic is sound and complete for the perfectible sequents, so this notion of relevance can be taken to underwrite Classical Core Logic as a relevance logic. ${ }^{19}$ Of course, I do not claim that the present notion of relevance is the only possible explication of relevance, so I am not claiming that Classical Core Logic is the 'right' relevance logic. But the notion of perfection provides a nice story for why it is a good one.

5.3. Smiley again. The second approach to entailment that Smiley considers is to define entailment in terms of proof, and he considers two such definitions. Under the first definition, $\phi_{1}, \ldots, \phi_{n}$ entail $\psi$ when there is a proof of $\psi$ from the $\phi_{i}$ 's, and under the second definition $\phi_{1}, \ldots, \phi_{n}$ entail $\psi$ when there is a proof of $\left(\phi_{1} \wedge \cdots \wedge \phi_{n}\right) \rightarrow \psi$. Each definition is given in the context of a deductive system that renders the Lewis argument invalid. The claim that every provably valid argument is an entailment is intuitive, ${ }^{20}$ but is only informative when we have some idea of what the correct axioms and rules of inference are. The difficulty is that all the rules involved in the deduction of the Lewis paradox are ex-

19 See Tennant (1984). Tennant (1987, p. 191) offers an argument similar to that in the main text.

20 Though Geach (1958) questions it. 
tremely compelling. And because Smiley defines a proof as simply a sequence of sentences each of which is either a premise, ${ }^{21}$ an axiom, or follows from earlier lines by a rule of inference, entailment is unrestrictedly transitive. So the Lewis paradox cannot be avoided by restricting transitivity, and hence requires either rejecting disjunction-introduction or disjunctive syllogism. As a result, Smiley ends up with awkward and gerrymandered sets of axioms and rules. ${ }^{22}$

The main technical difference between the two resulting concepts of entailment that Smiley discusses and the notions of relevance and perfection is that Smiley's definitions are both unrestrictedly transitive, whereas neither perfection nor relevance are. As a salient example, $A: A \vee B$ and $A \vee B, \neg A: B$ are both perfect (and hence relevant), but $A, \neg A: B$ is irrelevant. Other notable features are that in Smiley's first deductive system $A$ does not entail $A \vee B$, and in the second system modus ponens is not valid and $\wedge$ cannot be defined from $\neg$ and $\rightarrow$ in the usual way.

5.4. Anderson and Belnap. There are significant differences in both orientation and technical detail between my account of relevance and the systems in the tradition of Anderson and Belnap, ${ }^{23}$ but there are also some similarities underlying our different conceptions of relevance. The biggest difference is that the aim of the Anderson and Belnap tradition is to develop logics of relevance and entailment with an object-language connective expressing entailment. On my account, by contrast, the aim is not to develop a logic of relevance or perfection, but to study relevance and perfection in the context of classical logic. Accordingly, I feel no pressure to interpret the arrow as anything other than a material conditional. The fact that my project takes place in the context of classical logic and is not a project of logical revision also means that it will inherit the technical differences between classical logic and the Anderson-Belnap systems. Since these differences are well documented I will not discuss them here (see, e.g., Dunn \& Restall (2002)).

The similarity between Anderson and Belnap's account and my own comes in the underlying conception of relevance. They take it as a necessary and sufficient condition for $\phi$ to be relevant to $\psi$ that there be a derivation of $\psi$ that uses $\phi$ as an assumption. This is similar to the idea developed in $\S 2$ that a premise is relevant to an argument when it helps establish the validity of the argument; but instead of taking an argument to be a set of premises and a conclusion, this approach takes an argument to include the deduction that leads from the premises to the conclusion.

There are difficulties in developing this idea to motivate a particular relevance logic, though. Obviously, which premises can be used in a derivation of $\psi$ will depend on the other rules that allowed in the proof system, as Anderson and Belnap were well aware (Anderson \& Belnap, 1975, p. 31). For instance, if the proof system includes disjunctive syllogism and $\vee$-introduction, the Lewis argument will simply show that $A$ and $\neg A$ can be used in a derivation of $B$, and hence are relevant to $B$, intuition to the contrary notwithstanding (Bennett, 1965; Read, 1988). Or, given any deduction $\Pi$ of $\psi$ with premise $\theta$ (possibly among others), we can create a derivation of $\psi$ that seemingly uses $\phi$ as a premise as well:

21 Smiley does require that the premises actually be used in the derivation, so that irrelevant premises cannot be added.

22 This is a claim specifically about Smiley's systems. As an anonymous referee pointed out to me, not every logic that rejects one of disjunctive syllogism or disjunction introduction need be awkward and gerrymandered, as witnessed by FDE.

23 See Anderson \& Belnap (1975) and Anderson, Belnap, \& Dunn (1992); also Read (1988) and Dunn \& Restall (2002). 


\begin{tabular}{c}
$\frac{\phi \quad \theta}{\phi \wedge \theta}$ \\
\hline$\theta$ \\
$\Pi$ \\
$\psi$
\end{tabular}

So unless the deductive system precludes the standard $\wedge$ inferences, has normal-form requirements on proofs, or invokes a different notion of when a premise has been 'used', any premise will be relevant to any conclusion. There are ways of developing these replies, with the Anderson-Belnapians adopting a stricter notion of when a premise has been used that precludes this result (Dunn \& Restall, 2002, p. 23; incidentally, Tennant's systems achieve their relevance properties by a normal-form requirement on proofs). The point here is simply that understanding relevance as contributing to the validity of an argument, and in turn explicating that notion as use-in-a-deduction will not on its own suffice to weed out any arguments as irrelevant. Thus, despite the similarity between the Anderson-Belnap picture of relevance and my own, they must invoke additional restrictions to underwrite their systems of relevance logic.

Something similar is true of the other notion of relevance associated with the AndersonBelnap tradition, where:

the relevance [of $\phi$ to $\psi$ ] is now construed as involving some 'meaning content' common to both $[\phi]$ and $[\psi]$.... A formal condition for 'common meaning content' becomes almost obvious once we note that commonality of meaning in propositional logic is carried by commonality of propositional variables. (Anderson \& Belnap, 1975, pp. 32-33)

On this basis Anderson and Belnap suggest that some atom occurring in both $\phi$ and $\psi$ is a necessary condition for $\phi$ to be relevant to $\psi .^{24}$ This is merely a necessary condition, however, not a sufficient one. So again, this account of relevance is not on its own enough to underwrite the Anderson-Belnap systems of relevance logic. Given that neither of their formal conditions for relevance are sufficient to determine the properties of their preferred relevance logic(s), it is natural to wonder whether those conditions can really be taken to explicate the notion of relevance Anderson and Belnap were after.

5.5. Diaz. Diaz (1981) is remarkable in the literature on relevance logic for giving a precise account of what relevance is and tying his account to an informal notion of relevance more or less explicitly. The informal idea his account captures is that of an atom being essential to determining the truth or falsity of a formula in which it occurs. He sharpens this idea into two different accounts of relevance. The first, $t$-relevance, uses Kleene's strong three-valued truth tables, with the values interepred as true, false, and unknown. For a given $\phi$, a set of atoms $\left\{P_{1}, \ldots, P_{n}\right\}$ is truth-determining for $\phi$ if every truth-assignment that makes each $P_{i}$ either true or false (but may give other atoms the value unknown), makes $\phi$ either true or false. Then $\phi$ is $t$-relevant when no proper subset of the set of atoms that occur in $\phi$ is truth-determining for $\phi$. The intuitive idea is that if $P$ occurs

24 Although I do not take variable-sharing to be an explication of relevance- - the connection to any intuitive concept of relevance being tenuous at best-it is interesting to note that the perfect sequents of classical logic do enjoy a strong variable sharing property. This is a simple corollary of the variable sharing property that Tennant (2015) establishes for Classical Core Logic, since Classical Core Logic proves all classically perfect sequents. See Tennant's article for details. This result is also explained and proved in the companion to the present article. 
in $\phi$ and the set of atoms distinct from $P$ that occur in $\phi$ is truth-determining, then the truth-value of $P$ was irrelevant to determining the truth-value of $\phi$.

The second account of relevance Diaz develops builds on $t$-relevance by recognizing that not only can an atom occurring in $\phi$ be irrelevant to the truth-value of $\phi$, but so can a particular instance of an atom, even if another occurrence of that same atom is relevant. For instance, in $(A \vee \neg A) \vee(A \rightarrow B)$, the third instance of $A$ is irrelevant since the truth of $(A \vee \neg A) \vee(A \rightarrow B)$ is guaranteed by the first two occurrences of $A$. Diaz calls this concept $o$-relevance, and defines $\phi$ to be $o$-relevant if for every occurrence of an atom in $\phi$, if $\psi$ results from replacing that occurrence of that atom by a new atom, there is a two-valued truth assignment $v$ such that $v(\phi) \neq v(\psi)$. The intuitive idea is that $\phi$ is $o$ relevant when every single occurrence of every atom in $\phi$ has some bearing on the truth value of $\phi$.

The intuitive notion of relevance at play here is very similar to the idea I began with, of some proposition bearing on a question. I focused that question at the level of an argument, though, which led to our asking whether a premise bore on the validity of the argument. Diaz, by contrast, focuses on formulas rather than arguments, and asks whether an (occurrence of an) atom bears on the truth of the whole formula. Despite this difference in orientation, the nature of $\rightarrow$ in a system of $o$-relevance does allow us to compare $o$-relevance with my account. Diaz $(1987,116)$ proves that a tautology $\phi_{1} \rightarrow\left(\phi_{2} \rightarrow\left(\ldots\left(\phi_{n} \rightarrow \psi\right) \ldots\right)\right.$ is $o$-relevant just in case $\phi_{1}, \ldots, \phi_{n}: \psi$ is perfect and each of $\phi_{1}, \ldots \phi_{n}, \psi$ is $o$-relevant. It is easy to see that this is a stronger requirement than $\phi_{1}, \ldots, \phi_{n}: \psi$ being perfect, since $\emptyset:(A \vee \neg A) \vee B$ is perfect, but $(A \vee \neg A) \vee B$ is not $o$-relevant. So when we interpret $\rightarrow$ as entails, $o$-relevance is a stronger requirement than perfection. Under this same interpretation, $t$-relevance is easily be seen to be orthogonal to perfection and relevance (in my sense). For instance, $A: A \vee B$ is perfect, but $A \rightarrow(A \vee B)$ is not $t$-relevant, while $A: A \vee \neg A$ is irrelevant in my sense, but $A \rightarrow(A \vee \neg A)$ is $t$ relevant.

Interpreting $\rightarrow$ as entailment in a system of either $t$-relevance or $o$-relevance leads to an awkward concept of entailment, however (Diaz, 1981, Chap. 6-9), and perhaps this is not surprising. Diaz's definitions explicate the idea of the truth of a subformula being relevant to the truth of the whole formula, which, although related to the validity of an argument, is a distinct issue. So although it is instructive to observe these differences between $t$-relevance and $o$-relevance on the one hand and relevance and perfection on the other hand, not much significance should be given to these differences. We should simply be seen as focusing on different places that the concept of relevance applies in logic.

§6. Conclusion. We began this article with the Lewis paradox $A, \neg A \therefore B$ and the intuition that somehow this argument violated a standard of relevance. We wanted to find an account of relevance that would vindicate this intuition and that met the two criteria of having a clear connection to an intuitive concept of relevance, and of having clear significance for logic. Explicating the intuitive notion of a proposition bearing on a question led us to the formal notion of relevance in $\$ 2$ and its more demanding cousin perfection in $\S 3$. Both relevance and perfection vindicate the intuition that the Lewis paradox does flout the standard of relevance/perfection. By tracing the notion of bearing on the question of whether the conclusion is true to its formal counterpart of contributing to the validity of the argument, I have tried to show that there is a clear connection between the intuitive concept and the formal conditions of relevance and perfection that we ended up with. The logical significance of contributing to the validity of an argument is obvious, and, furthermore, I 
have argued that recognizing the interest of the concepts of relevance and perfection raises an interesting set of questions in proof theory.

I have focused here on how the notion of relevance applies in classical logic, but the present approach to relevance can also be extended to other logics. The extension to intuitionistic logic is relatively straightforward. If we restrict sequents to have either a singleton or the empty set on the right hand side, most everything that is said here can be taken over by the intuitionist. The extension to classical second-order logic is also relatively straightforward. Everything said here can be taken over to the second-order case. But in second-order logic we also have comprehension axioms (or rules, as they can also be formulated), and the logical strength of a valid sequent depends in part on the complexity of the instances of comprehension needed to prove the sequent as well as on the members of the premise and conclusion sets of that sequent. Just as premise sets can be unnecessarily strong to establish the conclusion of a sequent, comprehension axioms can be unnecessarily strong. So in extending the present account of relevance to second-order logic it would be natural to include some consideration of the comprehension axioms used to prove a particular sequent. Developing this idea further is outside the scope of the present work, though.

The situation is more complicated for substructural logics. ${ }^{25}$ Because of the wide variety of approaches to and interpretations of substructural logics, not much can be said in detail about how the present approach to relevance will apply to substructural logic in general. ${ }^{26}$ However, a few comments can be made about the general issues in applying the present notion of relevance to substructural logics. First, the consequence relation of some substructural logics can be interpreted in ways other than as truth-preservation. ${ }^{27}$ But the general concept of relevance that I have appealed to does not obviously make sense when consequence is understood as something other than truth-preservation. To apply the present notion of relevance in that context would seem to require some fundamental conceptual reorientation.

For substructural logics that are to be interpreted as truth-preserving, the essential ideas of the present approach to relevance will apply, but there will be technical questions that need to be addressed. The basic issue is to define a subsequent when the objects in the sequent are not sets. In some cases this will be straightforward; for instance, in a sequent calculus whose objects are multisets, we can say that $\Delta^{\prime}: \Phi^{\prime}$ is a subsequent of $\Delta: \Phi$ if there are some orderings of $\Delta, \Delta^{\prime}, \Phi$, and $\Phi^{\prime}$ such that $\Delta^{\prime}$ is an initial segment of $\Delta$ and $\Phi^{\prime}$ an initial segment of $\Phi$. Other cases, such as structures that have two different types of premise combination, are not as clear. Defining a subsequent would likely have to be done on a case-by-case basis. Another technical wrinkle is that many logics have distinct substructural presentations. For instance, the logic K3 based on Kleene's strong three-valued truth tables can be presented in a fairly standard set-based, two-sided sequent calculus, as well as in three-sided and four-sided sequent calculi (Shapiro, 2017). To apply the ideas of this article to such a context, we would want a notion of subsequent that is robust across the different calculi for a logic, so that we end up with a well defined notion of relevance. One way to address this problem would be to give the semantic notion of consequence priority, so that in the definitions above we write $\Delta \models \Phi$ in place of

25 Thanks to an anonymous referee for bringing this to my attention.

26 For an introduction, see Restall (2000).

27 For instance, linear logic can be given a computational interpretation, and the Lambek calculus is standardly interpreted as a theory of grammar (Abramsky, 1993; Lambek, 1958; Restall, 2000). 
$\Delta: \Phi$. For logics such as K3 that are primarily motivated by their semantic interpretation, this solution seems to me satisfactory. However, for logics such as $\mathrm{R}$ whose motivation lies primarily in their deductive properties - at least, the original motivation for $\mathrm{R}$ was its deductive properties - giving priority to semantic consequence is perhaps less natural. ${ }^{28}$

\section{BIBLIOGRAPHY}

Abramsky, S. (1993). Computational interpretations of linear logic. Theoretical Computer Science, 111, 3-57.

Anderson, A. R. \& Belnap, N. D. (1962). Tautological entailments. Philosophical Studies, 13(1), 9-24.

Anderson, A. R. \& Belnap, N. D. (1975). Entailment: The Logic of Relevance and Entailment, Vol. I. Princeton: Princeton University Press.

Anderson, A. R., Belnap, N. D., \& Dunn, J. M. (1992). Entailment: The Logic of Relevance and Entailment, Vol. II. Princeton: Princeton University Press.

Avron, A. (1984). Relevant entailment-Semantics and formal systems. Journal of Symbolic Logic, 49(2), 334-342.

Avron, A. (2014). What is a relevance logic? Annals of Pure and Applied Logic, 165, 2648.

Bennett, J. (1965). Review: T. J. Smiley, Entailment and Deducibility and Alan Ross Anderson and Nuel D. Belnap, The Calculus of Pure Entailment. Journal of Symbolic Logic, 30(2), 240-241.

Boolos, G., Burgess, J., \& Jeffrey, R. (2007). Computability and Logic (fifth edition). New York: Cambridge University Press.

Burgess, J. (1981). Relevance: A fallacy? Notre Dame Journal of Formal Logic, 22, 97 104.

Chang, C. \& Keisler, H. J. (2012). Model Theory (third edition). Mineola, NY: Dover.

Conan Doyle, A. (1976). The adventure of the priory school. In The Original Illustrated Sherlock Holmes. Edison, NJ: Castle Books, pp. 508-526.

Copeland, B. (1980). The trouble Anderson and Belnap have with relevance. Philosophical Studies, 37, 325-334.

Copeland, B. (1984). Horseshoe, hook, and relevance. Theoria, 50, 148-164.

Diaz, M. R. (1981). Topics in the Logic of Relevance. Munich: Philosophia Verlag.

Dunn, J. M. \& Restall, G. (2002). Relevance logic. In Gabbay, D. and Geunther, F., editors. Handbook of Philosophical Logic, Vol. 6. Dordrecht: Kluwer Academic, pp. 1-136.

Feferman, S. (1960). Arithmetization of metamathematics in a general setting. Fundamenta Mathematicae, 49(1), 35-92.

Frege, G. (1879). Begriffsschrift. In van Heijenoort, J., editor. From Frege to Gödel, Cambridge, MA: Harvard University Press, pp. 1-82.

Friedman, H. \& Flagg, R. C. (1990). A framework for measuring the complexity of mathematical concepts. Advances in Applied Mathematics, 11, 1-34.

Geach, P. (1958). Entailment. Proceedings of the Aristotelian Society, Supplementary Volumes, 32, 157-172.

Geach, P. (1970). Entailment. Philosophical Review, 79(2), 237-239.

28 Thanks to an audience at the 2017 Society for Exact Philosophy for helpful discussion when I presented this article, and to André Curtis-Trudel, Steven Dalglish, Teresa Kouri Kissel, Giorgio Sbardolini, Stewart Shapiro, Neil Tennant, and several referees for helpful comments on earlier drafts. 
Gemes, K. (2007). Irrelevance: Strengthening the bayesian requriements. Synthese, 157(2), 161-166.

Krämer, S. (2017). A hyperintensional criterion of irrelevance. Synthese, 194(8), 2917 2930.

Krämer, S. \& Roski, S. (2017). Difference-making grounds. Philosophical Studies, 174, $1191-1215$.

Lambek, J. (1958). The mathematics of sentence structure. American Mathematical Monthly, 65(3), 154-170.

Lapara, N. (1976). Semantics for a natural notion of entailment. Philosophical Studies, 29(2), 91-113.

Lehrer, K. (1973). Relevant deduction and minimally inconsistent sets. Philosophia, 3(3), $153-165$.

Lewis, C. I. \& Langford, C. H. (1959). Symbolic Logic. New York: Dover.

Makinson, D. (2017). Relevance via decomposition: A project, some results, an open question. Australasian Journal of Logic, 14(3), 356-377.

Mares, E. (2014). Relevance logic. In Zalta, E. N., editor. The Stanford Encyclopedia of Philosophy (Spring 2014 edition). Available at: https://plato.stanford. edu/archives/spr2014/entries/logic-relevance/.

Meyer, R. (1971). Entailment. Journal of Philosophy, 68(21), 808-818.

Prawitz, D. (2006). Natural Deduction: A Proof-Theoretical Study. Mineola, NY: Dover. Original published 1965.

Read, S. (1988). Relevant Logic. Oxford: Blackwell.

Restall, G. (2000). An Introduction to Substructural Logic. New York: Routledge.

Shapiro, L. (2017). Lp, k3, and fde as substructural logics. In Arazim, P. and Lavička, T., editors. The Logica Yearbook 2016. London: College Publications.

Smiley, T. (1959). Entailment and deducibility. Proceedings of the Aristotelian Society, 59, 233-254.

Tennant, N. (1979). Entailment and proofs. Proceedings of the Aristotelian Society, 79, 167-189.

Tennant, N. (1984). Perfect validity, entailment and paraconsistency. Studia Logica, 43, 179-198.

Tennant, N. (1987). Anti-Realism and Logic. Oxford: Clarendon Press.

Tennant, N. (2005). Relevance in reasoning. In Shapiro, S., editor. The Oxford Handbook of Philosophy of Logic and Mathematics. New York: Oxford University Press, pp. 696726.

Tennant, N. (2015). The relevance of premises to conclusions of core proofs. Review of Symbolic Logic, 8, 743-784.

Verdée, P. \& de Bal, I. (2015). A new approach to classical relevance. Studia Logica, 103, 919-954.

von Wright, G. (1957). The concept of entailment. In Logical Studies. London: Routledge and Kegan Paul, pp. 166-191.

Woods, J. (1964). Relevance. Logique et Analyse, 7(27), 130-137.

\section{DEPARTMENT OF PHILOSOPHY \\ THE OHIO STATE UNIVERSITY \\ 350 UNIVERSITY HALL 230 N. OVAL MALL \\ COLUMBUS, OH 43210, USA}

E-mail: eebrauer@gmail.com 